\title{
Il trattamento dei disturbi psicotici con olanzapina, risperidone e neurolettici tipici: una valutazione comparativa di costo/efficacia in una realtà psichiatrica locale

\author{
Egidio Filippelli*, Giovanni Biricolti§, Cinzia Scarano**, Federico Russo**, Lucio Luciano*
}

\begin{abstract}
BACKGROUND: Several clinical trials demonstrated that atypical antipsychotics are more effective but also more expensive (as drug cost) compared with the typical neuroleptics by treating psychotic disorders. The present study aimed to evaluate this result using an observational approach which better reflects the real clinical practice.

OBJECTIVE: To evaluate clinical effectiveness (including work and social functioning) and overall direct costs in a group of patients affected by psychotic disorders (schizophrenia and bipolar) and treated with typical and atypical (olanzapine and risperidone) antipsychotics.

METHODS: With a multicentre observational design - two years long - 89 patients (in charge by Psychiatric Centers of Regione Campania - Italy) were assessed using CGI (Clinical Global Impression) and GAF (Global Assessment of Functioning) scales. Moreover economic data were collected with reference to pharmacological and non-pharmacological (hospitalization, medical/nurse visits, etc.) resources consumption. The pharmacoeconomic analysis were conducted choosing the perspective of the local Psychiatric Services for costs attribution.

RESULTS: Considering the treatment outcomes, the use of the atypical drugs provided better performances with reference to the patients quality of life. The results in terms of work and social functioning indicated an advantage in the olanzapine group of patients. Overall direct costs of treatment (drugs and healthcare resources) didn't generate significant differences among the groups of therapy despite the pharmacological cost evidentiated an economic advantage $(\mathrm{p}<0,05)$ in the typical group due to the cheaper cost of these drugs. The use of olanzapine was associated to a lower number of hospitalizations and showed a general reduction ($16 \%$ ) of total treatment costs between 1st and 2 nd year of observation.

CONCLUSIONS: The lack of side effects, the improvement in work and social functioning, associated to a more efficient use of total healthcare resources seems to be at the basis of the better pharmacoeconomic profile for olanzapine compared with the other antipsychotic therapies.
\end{abstract}

Keywords: olanzapine, risperidone, typical neuroleptics, pharmacoeconomic analysis Farmeconomia e percorsi terapeutici 2005; 6 (3): 161-168

\section{INTRODUZIONE}

Gli antipsicotici atipici sono una scelta sicura ed efficace per il trattamento dei disturbi psicotici e della schizofrenia in particolare. Molti studi in doppio cieco hanno dimostrato i loro vantaggi clinici ed economici rispetto ai più tradizionali neurolettici tipici. Esistono anche ricerche che hanno comparato direttamente antipsicotici atipici tra di loro. Tuttavia la maggior parte di questi studi ha prodotto dati inconsistenti se trasferiti alla pratica clinica quotidiana, a causa dei limiti di selezione dei pazienti e della metodologia imposti dal disegno sperimentale in doppio cieco controllato, teso soprattutto a misurare differenze di efficacia e sicurezza [1,2].

Questa insufficienza descrittiva della realtà emerge soprattutto quando tra gli obiettivi di studio viene posta la valutazione economica dei trattamenti a confronto. Infatti la valutazione di efficacia e la valutazione economi-
*Dipartimento Salute Mentale ASL BN/1

$\S$ Health Outcomes Research - Eli Lilly Italia

**Area Farmaceutica ASL BN/1 
ca sono spesso in conflitto metodologico, avendo la prima necessità di operare su popolazioni di pazienti rigidamente selezionate, tanto da non poter essere facilmente rintracciabili in un contesto di quotidianità, mentre la seconda ha bisogno di un approccio più naturalistico per produrre valutazioni aderenti alla reale pratica clinica.

Per questo motivo si è ritenuto opportuno promuovere uno studio che seguisse un approccio naturalistico, senza prevedere strutturazioni di intervento e senza eccessive limitazioni in termini di selezione del campione. Seguendo questa impostazione sono stati analizzati i dati dell' attività svolta all'interno di alcune Strutture Psichiatriche della Regione Campania con lo scopo di confrontare costi ed efficacia associati al trattamento di pazienti psicotici in terapia con i nuovi farmaci "atipici" o con i "tipici" di vecchia generazione.

\section{OBIETTIVI E PROSPETTIVA DI ANALISI}

I nuovi antipsicotici atipici sono caratterizzati da un costo di acquisizione significativamente più alto dei tradizionali neurolettici tipici. Nell' ambito della presente analisi si vuole tuttavia focalizzare l'attenzione sui costi globali di trattamento dei pazienti psicotici che includono, oltre al costo per i farmaci, anche il ricorso alle prestazioni psichiatriche territoriali e ai ricoveri resi necessari durante le fasi di riacutizzazione della patologia.

In virtù di ciò l'obiettivo principale di questo studio è confrontare costi globali ed efficacia associati a due anni (2003-2004) di trattamento di pazienti psicotici in regime di terapia con farmaci atipici (olanzapina e risperidone) o neurolettici tipici e in carico presso alcune Strutture Psichiatriche della Regione Campania.

L'analisi costo/efficacia è stata condotta nella prospettiva della ASL e in particolare delle Strutture erogatrici delle prestazioni psichiatriche. In tal senso si sono considerati solo i costi di produzione ed erogazione dei trattamenti psichiatrici da parte delle Strutture pubbliche, nonché i soli farmaci a carico del SSN.

\begin{tabular}{llcc}
\hline \multicolumn{1}{c}{ Diagnosi } & \multicolumn{1}{c}{ Trattamento } & $\mathbf{N}^{\circ}$ pazienti & $\%$ \\
\hline Disturbo bipolare & Olanzapina & 3 & $37,5 \%$ \\
& Risperidone & 1 & $12,5 \%$ \\
& Tipici & 4 & $50,0 \%$ \\
Psicosi & Totale & 8 & $100 \%$ \\
schizofreniche & Olanzapina & 26 & $32,1 \%$ \\
& Risperidone & 29 & $35,8 \%$ \\
& Tipici & 26 & $32,1 \%$ \\
& Totale & 81 & $100 \%$ \\
\hline
\end{tabular}

Tabella I

Tipologia di trattamento attuato in relazione alla diagnosi rilevata

\section{METODOLOGIA}

La presente valutazione farmacoeconomica deriva da uno studio osservazionale multicentrico della durata di due anni condotto su 89 pazienti affetti da disturbi psicotici (schizofrenici e bipolari), in trattamento con farmaci tipici e atipici e seguiti dai Centri Psichiatrici di Benevento, Morcone e Puglianello appartenenti all'ASL BN/1.

L'osservazione è stata condotta, per l'anno 2003, in senso retrospettivo raccogliendo dati relativi alle prestazioni psichiatriche territoriali, alle ospedalizzazioni e al consumo di farmaci impiegati per il trattamento con olanzapina, risperidone o neurolettici tipici di pazienti psicotici in carico presso l'ASL BN/1.

I pazienti osservati nel 2003 sono stati poi seguiti, in senso prospettico, anche nel 2004 ove, oltre a raccogliere gli stessi dati di assorbimento di risorse sanitarie, si è proceduto anche a valutare l'outcome clinico associato ai diversi trattamenti attraverso la somministrazione delle scale di valutazione psichiatrica CGI (Clinical Global Impression) e GAF (Global Assessment of Functioning). I test di valutazione psichiatrica sono stati somministrati ai pazienti in corrispondenza della baseline (gennaio 04) e a tre successivi follow-up intervallati ciascuno da un periodo di quattro mesi (mag 04 - set 04 - dic 04).

Sono stati arruolati pazienti affetti da disturbi psicotici (diagnosi confermata secondo ICD9) e trattati con neurolettici tipici (30 pazienti), olanzapina (29 pazienti) e risperidone (30 pazienti). L'inclusione dei pazienti nei tre gruppi (olanzapina, risperidone, neurolettici tipici) è stata guidata dalla terapia antipsicotica principale che, in corrispondenza dell'arruolamento, doveva essere prescritta da almeno un anno, fase retrospettiva compresa.

Il campione osservato era costituito da 61 maschi e 28 femmine con un' età media di 41,5 anni (range 19-66).

Le diagnosi osservate secondo 1'ICD9 sono state: sindrome affettiva bipolare ( 8 pazienti) psicosi schizofreniche (81 pazienti). La Tabella I evidenzia il tipo di trattamento attuato in rapporto alle due diagnosi rilevate nello studio.

I pazienti osservati sono stati inseriti nei tre bracci di trattamento in modo che ciascun gruppo fosse confrontabile per età e gravità media della patologia. A tal proposito, non essendo stato possibile ricostruire retrospettivamente la gravità clinica dei pazienti, si è posto attenzione al fatto che il numero medio per paziente di episodi di ricovero nell'anno precedente l'inizio dell'osservazione retrospettiva (ovvero nell' anno 2002) fosse confrontabile fra i tre gruppi (Tabella II). 
Dal momento che l'efficacia dei trattamenti è stata misurata anche con riferimento alla loro capacità di ri-avviamento all'attività lavorativa, si è posta particolare attenzione alla condizione lavorativa dei pazienti nei tre bracci di trattamento, verificando la sovrapponibilità nei gruppi delle categorie sociali "lavoratore", "disabile", "pensionato", "disoccupato", etc.

Inoltre, sempre per garantire la massima confrontabilità fra i tre gruppi, nel braccio "neurolettici tipici" non sono stati inseriti pazienti in terapia principale con formulazioni depot. La mancanza infatti di formulazioni long-acting per gli antipsicotici atipici ha obbligato necessariamente il confronto sul versante delle formulazioni orali ad assunzione quotidiana, al fine di non alterare il peso della variabile compliance.

\section{TIPOLOGIA DI DATI RACCOLTI}

Nel corso dei due anni di osservazione del presente lavoro sono stati raccolti dati riguardanti sia l'efficacia che i costi totali di trattamento dei pazienti psicotici associati alle diverse strategie terapeutiche poste a confronto.

Sul versante economico si è focalizzata l'attenzione sulla misurazione dei costi diretti, relativi cioè al consumo da parte del paziente psicotico di tutte le prestazioni sanitarie attribuibili direttamente alla patologia. A tal riguardo si è proceduto a raccogliere, in entrambi gli anni di osservazione, dati sui consumi di farmaci per il trattamento della patologia psichiatrica (antipsicotici principale ed associati, antidepressivi, anticolinergici, stabilizzatori dell'umore), sulle ospedalizzazioni e sulle prestazioni psichiatriche territoriali quali: a) visite specialistiche, b) interventi infermieristici domiciliari e in struttura, c) interventi medici domiciliari, d) interventi di psicoterapia.

Coerentemente con la prospettiva di analisi di questo lavoro, la valorizzazione dei consumi di risorse sanitarie da parte dei pazienti psicotici è stata effettuata utilizzando i costi reali di erogazione delle prestazioni sostenuti dalle Strutture Psichiatriche pubbliche. Tali costi sono stati tratti da alcune recenti pubblicazioni a carattere psichiatrico e attualizzati all'anno $2005[3,4]$. La valutazione dei costi ospedalieri è stata effettuata utilizzando i dati di costo per giornata di ricovero in SPDC (Servizio Psichiatrico Diagnosi e Cura).

I consumi farmacologici sono stati valorizzati utilizzando i prezzi riportati dall' Informatore Farmaceutico Italiano, anno 2004.

Sul piano dell'efficacia l'outcome associato ai diversi trattamenti è stato misurato utilizzando la variabile ricoveri durante la fase retrospettiva dello studio (anno 2003) mentre in sede di valutazione prospettica (anno 2004) i risultati associati alle diverse terapie sono scaturiti dalla

\begin{tabular}{cccc}
\hline & $\mathbf{N}^{\circ}$ pazienti & $\begin{array}{c}\mathbf{N}^{\circ} \text { episodi di } \\
\text { ricovero 2002 }\end{array}$ & $\begin{array}{c}\mathbf{N}^{\circ} \text { medio di ricoveri } \\
\text { a paziente 2002 }\end{array}$ \\
\hline Olanzapina & 29 & 5 & 0,17 \\
Risperidone & 30 & 4 & 0,13 \\
Tipici & 30 & 4 & 0,13 \\
\hline
\end{tabular}

Tabella II

Numero medio a paziente di episodi ricovero per tipo di trattamento nell'anno 2002

somministrazione ai pazienti delle scale CGI (Clinical Global Impression - \#028) e GAF (Global Assessment of Functioning - \#320) ad intervalli di tempo di quattro mesi ciascuno, al fine di integrare i dati di valutazione clinica (CGI) con quelli di funzionamento sociale (GAF) [5].

Durante entrambi gli anni di osservazione è stata inoltre valutata anche la capacità di ciascun trattamento di ripristinare le normali funzioni psico-fisiche dei pazienti, misurando in particolare il loro re-inserimento nell' ambiente lavorativo o addirittura il ritorno ad attività di studio (università, corsi professionali, etc.). Il parametro con cui si sono valutati questi risultati è rappresentato dal numero medio annuo per paziente di giornate dedicate all'attività lavorativa/studio. Esso è sintomatico non solo della qualità della vita associata a ciascun trattamento ma anche del miglioramento del quadro clinico del paziente espresso al suo re-inserimento nel "tessuto sociale".

\section{RISULTATI}

\section{Valutazione dell'efficacia}

L'analisi della Tabella III ci permette di rilevare una riduzione della gravità alla CGI in tutti e tre i bracci nel corso del 2004 con una significatività statistica nel gruppo atipici (olanzapina $\mathrm{p}<0,001$; risperidone $\mathrm{p}=0,018$ ).

\begin{tabular}{ccccc}
\hline $\begin{array}{c}\text { CGI 2004 } \\
\text { (gravità) }\end{array}$ & t0 2004 & t3 2004 & Diff. & p value \\
\hline Olanzapina & 3,83 & 3,48 & $-0,35$ & $<0,001$ \\
Risperidone & 4,00 & 3,73 & $-0,27$ & 0,018 \\
Tipici & 4,00 & 3,93 & $-0,07$ & 0,423 \\
\hline
\end{tabular}

\section{Tabella III}

Punteggio medio gravità CGI per tipo di terapia: differenze fra baseline e III follow-up

\begin{tabular}{ccccc}
\hline GAF 2004 & t0 2004 & t3 2004 & Diff. & p value \\
\hline Olanzapina & 62,24 & 65,90 & 3,66 & 0,036 \\
Risperidone & 59,57 & 61,57 & 2,00 & 0,121 \\
Tipici & 62,40 & 64,80 & 2,40 & 0,038 \\
\hline
\end{tabular}

Tabella IV

Punteggio medio GAF per tipo di terapia: differenze fra baseline e III follow-up 


\begin{tabular}{cccc|ccc}
\hline CGI 2004 & \multicolumn{3}{c}{$\begin{array}{c}\text { Gravità medio-alta } \\
\text { moderata-marcata-grave }\end{array}$} & \multicolumn{3}{c}{$\begin{array}{c}\text { Gravità bassa } \\
\text { lieve-molto lieve }\end{array}$} \\
\cline { 2 - 7 } & t0 2004 & t3 2004 & Diff. & t0 2004 & t3 2004 & Diff. \\
\hline Olanzapina & 22 & 12 & -10 & 7 & 17 & 10 \\
Risperidone & 23 & 20 & -3 & 7 & 10 & 3 \\
Tipici & 25 & 24 & -1 & 5 & 6 & 1 \\
\hline
\end{tabular}

\section{Tabella V}

Distribuzione pazienti per fasce di gravità CGI e per tipo di trattamento: differenze fra baseline e III follow-up

La significatività statistica del miglioramento rilevato con la GAF nel 2004 la si riscontra nei gruppi olanzapina $(\mathrm{p}=0,036)$ e in quello trattato con tipici $(\mathrm{p}=0,038)$ (Tabella IV).

Sono stati inoltre raggruppati gli item di gravità della CGI in due cluster: gravità medio/alta (moderata, marcata, grave) e gravità bassa (lieve, molto lieve). Nel primo cluster al t0 si rileva-

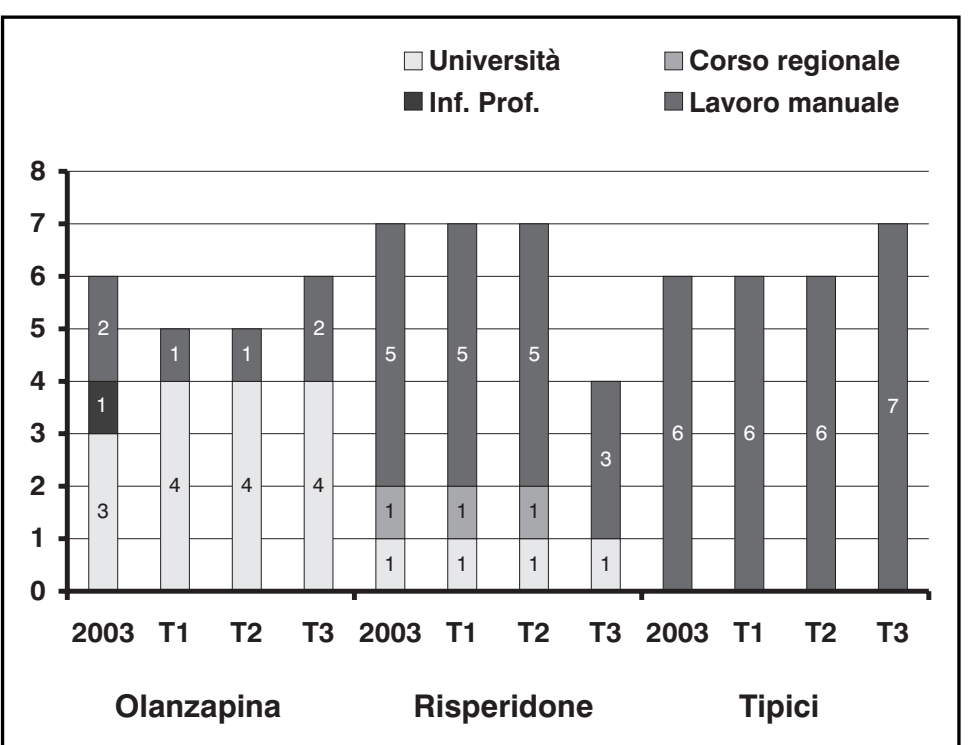

\section{Figura 1}

Numero di pazienti che hanno svolto attività lavorativa/studio nel biennio 200304 per tipo di terapia

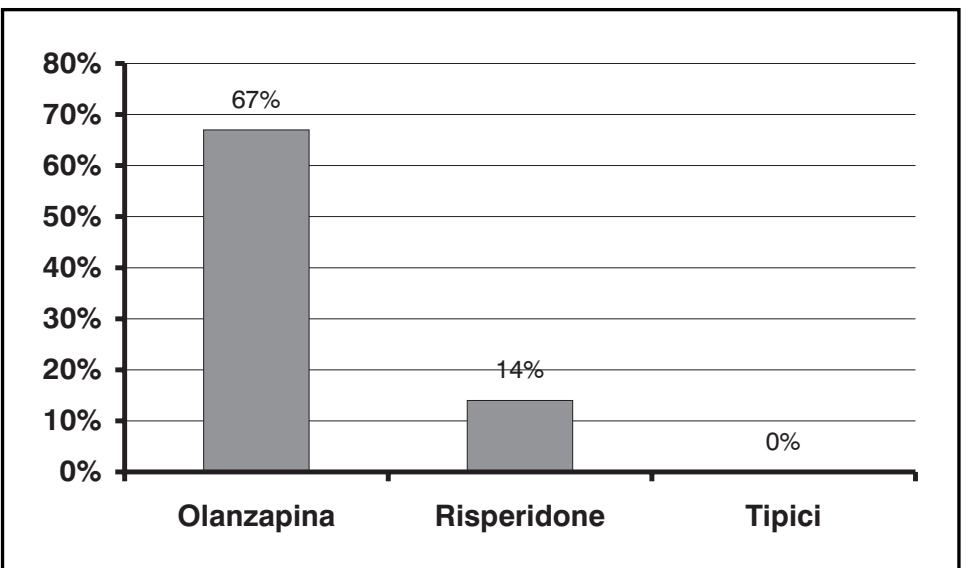

Figura 2

Percentuale media di universitari su totale pazienti attivi nel biennio 2003-04 per tipo di terapia vano 22 pazienti trattati con olanzapina, 23 con risperidone e 25 con tipici; nel secondo cluster 7 ad olanzapina, 7 a risperidone e 5 a tipici.

Alla valutazione finale dello studio $\mathrm{t} 3$ si rilevava la seguente situazione circa il passagio dei pazienti dalla prima alla seconda fascia (gravità medio/alta > gravità bassa): olanzapina 10 pazienti, risperidone 3 pazienti, tipici 1 paziente (Tabella V).

La valutazione del funzionamento sociale acquista un particolare valore alla luce degli altissimi costi sostenuti per il trattamento di una patologia devastante quale quella schizofrenica. In una recente analisi si è stimato che circa il $70 \%$ di tali costi è rappresentato da quelli indiretti: perdita di produttività, interventi assistenziali da parte dello stato, carico familiare [6].

A tal proposito il nostro studio ha evidenziato che circa l' $84 \%$ dei pazienti osservati (89) non è stato in grado di svolgere alcun tipo di attività lavorativa o di studio durante il biennio di osservazione. Del 16\% (19 pazienti) che ha invece svolto attività lavorativa nel 2003, 6 erano presenti nel braccio olanzapina, 7 nel braccio risperidone, 6 nel braccio tipici. A fine osservazione (t3 2004) 6 erano nel braccio olanzapina, 4 nel braccio risperidone, 7 nel braccio tipici (Figura 1).

Si è riscontrato inoltre che l'attività lavorativa associata ai pazienti inseriti nel braccio tipici è stata esclusivamente di tipo manuale.

Nella valutazione dell'attività lavorativa abbiamo incluso la frequenza di corsi di studi, anche di tipo universitario. La percentuale media di universitari sul totale di pazienti attivi nel biennio di osservazione è stata la seguente: $67 \%$ nel gruppo olanzapina; $14 \%$ nel gruppo risperidone; 0\% nel gruppo tipici (Figura 2). Probabilmente ciò è indice del diverso impatto sul funzionamento cognitivo degli antipsicotici confrontati.

Per frequenza di un corso universitario si è inteso la partecipazione alle attività didattiche e il superamento degli esami da parte dei pazienti. A tal riguardo nel gruppo universitari trattati con olanzapina il $70 \%$ era prossimo alla conclusione del corso di studi (meno di 4 esami alla laurea).

\section{Valutazione dei costi}

Numerosi studi clinici hanno dimostrato che l'efficacia degli antipsicotici atipici è nettamente superiore rispetto a quella dei neurolettici tradizionali nel ridurre i sintomi positivi e negativi della schizofrenia e nel determinare minori effetti collaterali severi [7]. A fronte di questa maggiore efficacia, gli atipici presentano tuttavia un costo di acquisto notevolmente superiore.

La Tabella VI mostra i dati sui costi per farmaci rilevati nel corso dell'osservazione prospettica (anno 2004). 


\begin{tabular}{|c|c|c|c|c|c|}
\hline & Neurolettici & Antidepressivi & Anticolinergici & $\begin{array}{c}\text { Stabilizzatori } \\
\text { Umore }\end{array}$ & $\begin{array}{c}\text { Totale } \\
\text { Farmaci }\end{array}$ \\
\hline Olanzapina & 2.348 & 53 & 1 & 65 & 2.467 \\
\hline Risperidone & 1.388 & 18 & 1 & 19 & 1.426 \\
\hline Tipici & 226 & 27 & 1 & 18 & 272 \\
\hline \multicolumn{6}{|l|}{ Significatività } \\
\hline O-R & $<0,0001$ & ns & ns & ns & $<0,0001$ \\
\hline O-T & $<0,0001$ & ns & ns & ns & $<0,0001$ \\
\hline R-T & $<0,0001$ & ns & ns & ns & $<0,0001$ \\
\hline
\end{tabular}

Tabella VI

Costi farmacologici medi annui a paziente per tipo di terapia (anno 2004-dati in euro)
La Figura 3 riporta invece le variazioni di costo farmacologico per tipo di terapia riscontrate nell'anno 2004 rispetto al 2003.

Probabilmente il miglioramento delle condizioni psicopatologiche dei pazienti trattati con atipici ha consentito l'utilizzo di un minor dosaggio degli stessi nel 2004 rispetto al 2003 e quindi una riduzione dei relativi costi. Il dato sui tipici riflette invece probabilmente la necessità di variazioni, in aumento, del dosaggio o di associazioni con altri farmaci.

La diminuzione dei costi totali di terapia farmacologica per gli atipici è avvenuta nonostante l'associazione con farmaci complessi quali gli stabilizzanti dell'umore. La possibilità di associare olanzapina a molecole quali il litio, il valproato, la carbamazepina ci porta a due ordine di considerazioni: a) uso di olanzapina in condizioni cliniche complesse; b) maneggevolezza della molecola in associazione con farmaci di non facile gestione.

La valutazione dei costi degli interventi sanitari per tipo di trattamento fa rilevare, nel 2004, un risparmio di circa il $70 \%$ nel gruppo olanzapina rispetto ai tipici e di circa il $40 \%$ di risperidone rispetto ai tipici (Figura 4).

Questo fenomeno è attribuibile principalmente alla variabile ricoveri, come evidenziato in Tabella VII.

Il numero medio annuo a paziente di giornate di ricovero si è azzerato per olanzapina nel 2004, mentre invece ha mostrato un trend in crescita per risperidone e tipici come dimostrato dal relativo andamento dei costi in Figura 5.

Il mix di costo totale medio annuo di trattamento per il paziente psicotico rilevato nel biennio 2003-04 è gravato dalla voce ricoveri per il $72 \%$ nel gruppo tipici, per il 50\% nel gruppo risperidone e solo per il $4 \%$ nel gruppo olanzapina (Figura 6).

La valutazione dei costi globali di trattamento (farmaci+interventi sanitari) mostra in conclusione che il maggior costo per farmaci rilevato nel gruppo atipici è più che bilanciato dal minor ricorso all'utilizzo di altre risorse sanitarie (ricoveri in primis) rispetto ai neurolettici tipici. I dati evidenziati in Tabella VIII mostrano differenze non significative fra le terapie in termini di costi totali di trattamento, nonostante si rilevi un trend positivo in favore degli atipici.

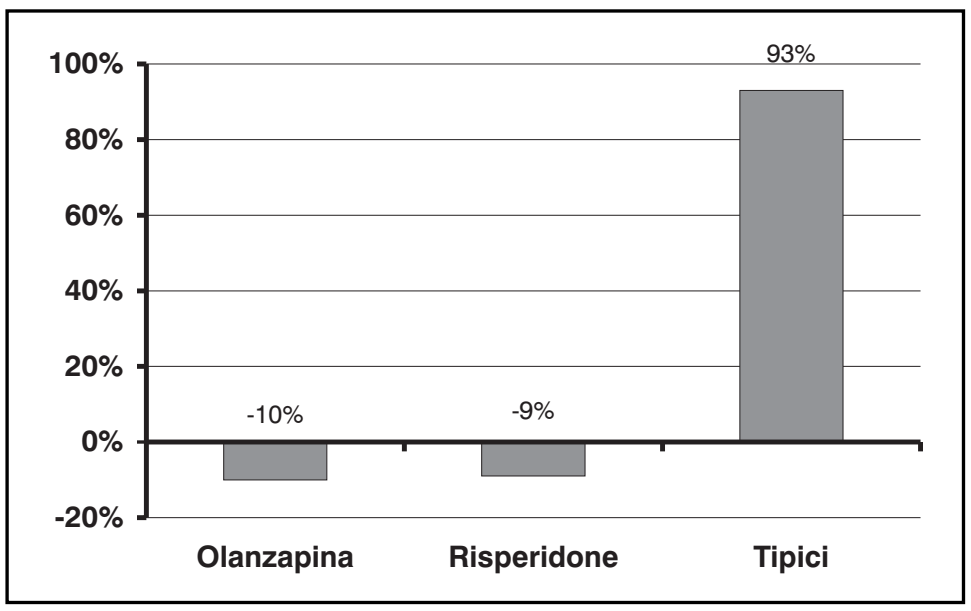

Figura 3

Variazione percentuale costo terapia farmacologica 2004 vs 2003 per tipo di trattamento

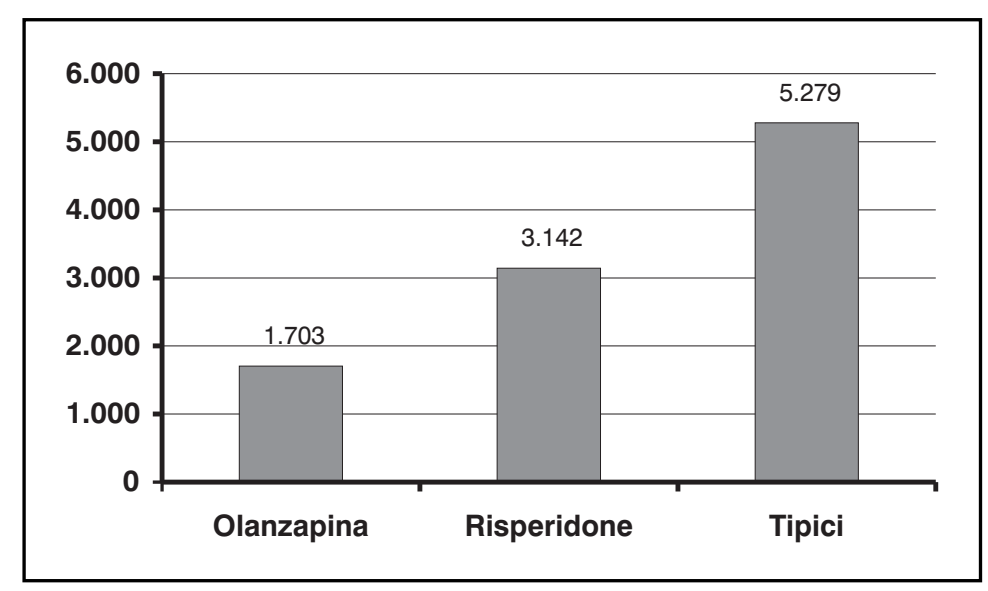

Figura 4

Costo medio a paziente per interventi sanitari non farmacologici per tipo di terapia (anno 2004-dati in euro)

\begin{tabular}{ccc|cc}
\hline & \multicolumn{2}{c}{2003} & \multicolumn{2}{c}{2004} \\
\cline { 2 - 5 } & $\begin{array}{c}\mathbf{n}^{\circ} \text { gg. di } \\
\text { ricovero }\end{array}$ & $\begin{array}{l}\text { costo } \\
\text { (euro) }\end{array}$ & $\begin{array}{c}\mathbf{n}^{\circ} \text { gg. di } \\
\text { ricovero }\end{array}$ & $\begin{array}{c}\text { costo } \\
\text { (euro) }\end{array}$ \\
\hline Olanzapina & 1,2 & 336 & 0,0 & 0 \\
Risperidone & 7,3 & 2.102 & 8,5 & 2.437 \\
Tipici & 17,3 & 2.764 & 21,7 & 4.059 \\
\hline
\end{tabular}

\section{Tabella VII}

Numero medio annuo di giorni di ricovero a paziente per tipo di terapia e relativi costi (biennio 2003-04) 


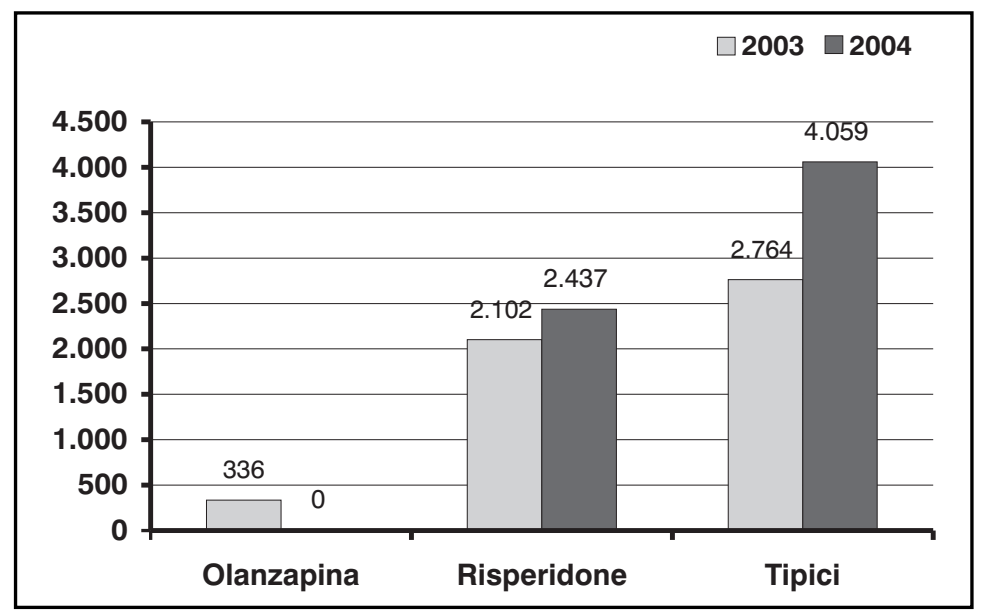

Figura 5

Trend costo medio annuo a paziente per giornate di ricovero per tipo di terapia (biennio 2003-04-dati in euro)

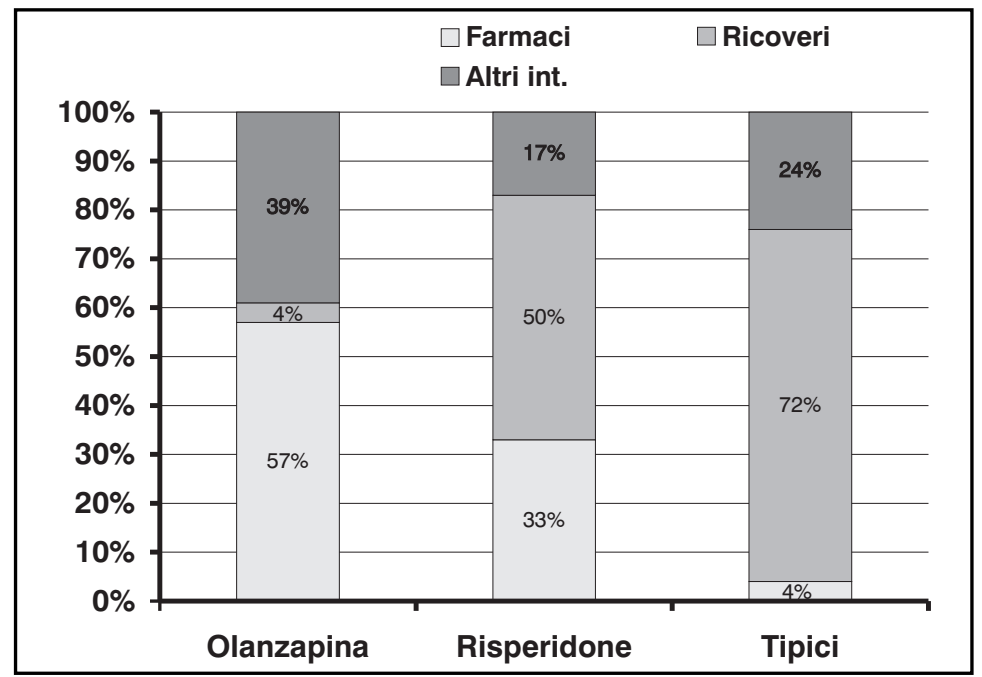

Figura 6

Mix costo totale medio annuo di trattamento per paziente psicotico nel biennio 2003-04 per tipo di terapia (dati in euro)

\begin{tabular}{lccc}
\hline & Farmaci & $\begin{array}{c}\text { Interventi } \\
\text { Sanitari }\end{array}$ & Totale \\
\hline Olanzapina & 2.467 & 1.703 & 4.170 \\
Risperidone & 1.426 & 3.142 & 4.568 \\
Tipici & 272 & 5.279 & 5.551 \\
Significatività & & & \\
O-R & $<0,0001$ & ns & ns \\
O-T & $<0,0001$ & ns & ns \\
R-T & $<0,0001$ & ns & ns \\
\hline
\end{tabular}

Tabella VIII

Costi totali medi annui a paziente per tipo di terapia (anno 2004-dati in euro)

\section{Analisi costo/efficacia}

Quando la valutazione economica non si ferma unicamente al versante dei costi ma si spinge anche a quello dei risultati, la farmacoeconomia suggerisce l'utilizzo della tecnica denominata analisi di costo/efficacia [8].
Spesso in ambito sanitario la limitata disponibilità di risorse impone di operare delle scelte tra programmi terapeutici alternativi. In questo caso si ricorre frequentemente al calcolo del rapporto medio costo/efficacia, e si ordinano i diversi programmi in base al rapporto medio partendo da quelli con il valore più basso fino ad arrivare a quelli con il valore più alto.

Sulla base delle risorse a disposizione (budget) si procederà quindi alla selezione del programma più efficiente ovvero che garantisca il miglior risultato di efficacia al costo più basso.

Nelle Tabelle IX e X abbiamo inserito i costi totali medi di trattamento del paziente psicotico (2004) rapportandoli ai risultati di efficacia rilevati per ciascuna terapia a confronto sulle scale psichiatriche CGI e GAF, calcolando così il "costo medio per il passaggio di un paziente da fasce di medio/alta gravità a fasce di bassa gravità CGI" e il "costo medio per punto di gravità ridotto su scala GAF".

\section{DISCUSSIONE}

In questo studio abbiamo deciso di adottare un approccio di tipo osservazionale su pazienti normalmente afferenti a Strutture Psichiatriche territoriali al fine di ottenere una valutazione farmacoeconomica del trattamento dei disturbi psicotici il più possibile aderente alla reale pratica clinica.

Il disturbo psicotico rappresenta da sempre un problema di complessa gestione per gli operatori sanitari e per la Società in generale, essendo caratterizzato da un lungo decorso, con fasi di riesacerbazioni che riducono significativamente la consapevolezza di malattia e l'aderenza alla terapia (compliance).

Per tali motivi è stato valutato il consumo di risorse sanitarie da parte del paziente psicotico includendo nell'analisi visite specialistiche, interventi medici domiciliari, interventi infermieristici domiciliari e in struttura, ricoveri, interventi di psicoterapia, oltre al costo di acquisto dei farmaci.

La misurazione dell' efficacia dei trattamenti è stata invece effettuata utilizzando le scale psichiatriche CGI (Clinical Global Impression) e GAF (Global Assessment of Functioning). Esse sono tra loro complementari nell'ottica di un giudizio complessivo sul paziente psicotico in quanto la prima consente una generale valutazione clinica, mentre la seconda è più orientata ad una rilevazione del funzionamento sociale.

La scarsa numerosità del campione osservato (89 pazienti) unita alla non-inclusione nello spettro di analisi di altre terapie antipsicotiche potrebbero costituire potenziali bias del no- 
stro lavoro fornendo un quadro non completo di valutazione economica del trattamento dei disturbi psicotici.

In ogni caso la valutazione globale dei costi di trattamento (farmaci + risorse sanitarie) ha evidenziato i seguenti valori per ciascun tipo di terapia:

- gruppo olanzapina: costo totale medio annuo per paziente (2004) pari a 4.170 euro;

- gruppo risperidone: costo totale medio annuo per paziente (2004) pari a 4.568 euro;

- gruppo neurolettici tipici: costo totale medio annuo per paziente (2004) pari a 5.551 euro.

La Tabella XI completa tali dati con l'indicazione degli scostamenti percentuali sui costi totali associati alle terapie e rilevati tra il 2003 e il 2004.

Questi risultati stanno ad indicare che il costo di acquisto del farmaco è solo una componente del costo totale sostenuto dall'ASL per il trattamento di una patologia cronica quale la schizofrenia. I dati dimostrano che, con l'utilizzo degli atipici, al maggior costo di acquisto del farmaco corrisponde un minor consumo delle altre risorse sanitarie. Questo è da attribuire probabilmente ad un uso più precoce degli atipici in tali patologie (farmaci di prima linea) e ad una loro maggiore efficacia.

La Tabella XI evidenzia olanzapina come il farmaco con il miglior profilo economico. In termini di efficacia la stessa molecola è l'unica in grado di ridurre, in maniera statisticamente significativa, il punteggio di gravità su scala CGI e di ottenere contemporaneamente un miglioramento del funzionamento globale alla GAF (Tabelle III e IV), con indubbie positive ripercussioni sulla qualità della vita. Nel gruppo olanzapina infatti ben il $67 \%$ dei pazienti con attività lavorativa ha riscontrato una fre-

\begin{tabular}{cccc}
\hline & $\begin{array}{c}\text { Costo totale } \\
\text { medio annuo } \\
\text { di trattamento }\end{array}$ & $\begin{array}{c}\text { Numero di pazienti } \\
\text { da medio/alta a } \\
\text { bassa gravità CGI }\end{array}$ & $\begin{array}{c}\text { Costo medio per } \\
\text { spostare un paziente } \\
\text { da medio/alta } \\
\text { a bassa gravità CGI }\end{array}$ \\
\hline Olanzapina & 4.170 & 10 & 417 \\
Risperidone & 4.568 & 3 & 1.523 \\
Tipici & 5.551 & 1 & 5.551 \\
\hline
\end{tabular}

Tabella IX

Costo medio per il passaggio di un paziente da fasce di medio/alta gravità a fasce di bassa gravità CGI (dati in euro)

\begin{tabular}{cccc}
\hline & $\begin{array}{c}\text { Costo totale } \\
\text { medio annuo } \\
\text { di trattamento }\end{array}$ & $\begin{array}{c}\text { Differenza di } \\
\text { punteggio GAF tra } \\
\text { rilevazioni t0-t3 }\end{array}$ & $\begin{array}{c}\text { Costo medio per } \\
\text { punto di gravità } \\
\text { ridotto su scala GAF }\end{array}$ \\
\hline Olanzapina & 4.170 & 3,66 & 1.139 \\
Risperidone & 4.568 & 2,00 & 2.284 \\
Tipici & 5.551 & 2,40 & 2.313 \\
\hline
\end{tabular}

Tabella $X$

Costo medio per punto di gravità ridotto su scala GAF (dati in euro)

\begin{tabular}{ccc}
\hline & $\begin{array}{c}\text { Costi totali } \\
\text { medi annui a paziente }\end{array}$ & $\begin{array}{c}\text { Variazione \% } \\
\mathbf{2 0 0 4} \text { vs 2003 }\end{array}$ \\
\hline Olanzapina & 4.170 & $-16 \%$ \\
Risperidone & 4.568 & $1 \%$ \\
Tipici & 5.551 & $41 \%$ \\
\hline
\end{tabular}

Tabella XI

Costi totali medi annui per paziente per tipo di terapia 2004 e variazioni percentuali su 2003 (dati in euro)

quenza attiva e documentata di corsi universitari (Figura 2).

La scarsità di effetti collaterali, il miglioramento del funzionamento sociale e cognitivo, la maneggevolezza uniti all' affidabilità sul piano economico sembrerebbero alla base del miglior profilo di costo/efficacia per olanzapina.

\section{BIBLIOGRAFIA}

1. Sturm R, Bao Y. Datapoints: psychiatric care expenditures and length of stay: trends in industrialized countries. Psychiatr. Serv.; 2000; 51,295

2. Sturm R, Unutzer J, Katon W. Effectiveness research and implications for study design: sample size, and statistical power; Gen. Hosp. Psychiatry; 1999; 21, 274-283

3. Garattini L. et al. ASI, 1996, n.10:33-37

4. Mapelli V. et al. Costi ed efficacia della cura della schizofrenia con antipsicotici tipici ed atipici, PharmacoEconomics Italian Research Articles, 2003; vol. 5, n. 3, 1-15

5. Conti L. Repertorio delle scale di valutazione in Psichiatria, tomo primo; See-Firenze; 1999; 197-207 
6. Wyatt RJ, Henter I, Leary MC, Taylor E. An economic evaluation of schizophrenia; Soc. Psychiatry Psychiatr. Epidemiol.; 1991; 30, 196-205

7. Duggan L, Fenton M, Dardennes RM, El-Dosoky A, Indran S. Olanzapine for schizophrenia; Cochrane Library (1); 2000; $1-81$

8. Tarricone R. Valutazioni economiche e management in sanità: applicazioni ai programmi e tecnologie sanitarie; Collana Management e Sanità; Mc Graw Hill, 2004

9. Fattore G. et al. I costi delle prestazioni in psichiatria, Epidemiologia e Psichiatria Sociale, 1997, 6(2): 139-147

10. L'Informatore farmaceutico 2004. OEMF, 65a edizione

11. Rossi I, Guidi L. Valutazione dei costi di trattamento dei disturbi psicotici con olanzapina, risperidone e neurolettici tipici di un DSM italiano, Farmeconomia e percorsi terapeutici, 2001; 2 (4): 253-258

12. Vadruccio F, Biricolti G, Mendolicchio L. Analisi farmacoeconomica del trattamento di pazienti psicotici cronici con olanzapina, antipsicotici atipici e neurolettici tipici: uno studio regionale; Farmeconomia e percorsi terapeutici; 2005; 6(2) 97-104 